Boletín de la Sociedad Geológica Mexicana

VOLUMEN 61, NÚM. 2, 2009, P. 167-175

\title{
Uso de las Propiedades Fisicoquímicas de Oligonucleótidos como Biomarcadores
}

\author{
José Martínez Reyes ${ }^{1}$, Eduardo González Partida ${ }^{1}$, Renee J. Perez ${ }^{2}$ \\ ${ }^{1}$ Centro de Geociencias, Universidad Nacional Autónoma de México, Campo de Juriquilla, Qro., México, apartado postal 76230. \\ ${ }^{2}$ Department of Chemical and Petroleum Engineering, University of Calgary, 500 University Drive, Calgary Alberta, Canada, T2N \\ 1N4. \\ *jmreyes@geociencias.unam.mx
}

\begin{abstract}
Resumen
Marcadores biológicos (biomarcadores) son complejos moleculares que pueden estar presentes en fósiles, procedentes de biomoleculas de los organismos vivos. Debido a sus características generales son resistentes a la intemperie, la biodegradación, la evaporación y otros procesos biológicos, siendo comúnmente conservados en las rocas y pueden ser utilizados por geólogos, geoquímicos, arqueólogos, etc. para obtener información sobre la materia orgánica en las rocas fuente, la presencia de petróleo, las condiciones ambientales durante su sedimentación (diagénesis), la madurez térmica experimentada por el petróleo y / o roca (catagénesis), el grado de biodegradación, algunos aspectos de la mineralogía de la roca fuente (litología), la edad de los fósiles y en caracterización de DNA (Acido Desoxirribonucleico). Los biomarcadores pueden ser oligonucleótidos, que son fragmentos de moléculas de DNA de cadena sencilla de determinada longitud.

El DNA de fósiles puede formar enlaces cruzados entre si o con otras moléculas al paso del tiempo, dificultando el uso de técnicas como el PCR para su estudio, por ello, en el presente trabajo se aplicó a los oligonucleótidos la técnica de determinación de los valores de $\mathrm{pK}_{\mathrm{a}}$ (potencial de la constante de ionización), mediante espectrofotometría de luz ultravioleta en el intervalo básico de $\mathrm{pH}$, usando como oligonucleótido modelo el d-AAAGAAA en solución acuosa de $\mathrm{NaCl}$, titulándolo con solución de $\mathrm{NaOH}$. Lo anterior también se realizo en presencia de una solución amortiguadora de $\mathrm{pH}\left(\mathrm{NaHCO}_{3}\right)$. Además se determinaron valores de $\mathrm{pK}_{\mathrm{a}}$ para el mismo compuesto a diferentes condiciones de temperatura, sales y mezclas de alcohol-agua como solvente. También se determinaron valores de $\mathrm{pK}_{\mathrm{a}}$ a los oligonucleótidos d-CCCGCCC y d-AAGAA.

Los valores de $\mathrm{pK}_{\mathrm{a}}$ obtenidos varían entre 9 y 12. Estos resultados obtenidos son un aporte al conocimiento de las propiedades físicas de los oligonucleótidos, estos datos pueden ser especialmente útiles en el mencionado caso de dificultad para el uso de otras técnicas como el PCR para la caracterización e interpretación de los oligonucleótidos como biomarcadores.
\end{abstract}

Palabras clave: oligonucleótidos, biomarcadores, constantes de ionización.

\begin{abstract}
Biological markers (biomarkers) are complex molecular fossils from biomolecules in living organisms. Due to their general are resistant to weather, the biodegradation, evaporation and other biological processes, as commonly preserved in rocks and can be used by geologists, geochemists, archaeologists, etc. for information on organic matter in source rocks, the presence of oil, environmental conditions during sedimentation (diagenetic process), the thermal maturity experienced by the oil and / or rock (catagenetic process), the degree of biodegradation, some aspects of mineralogy of the source rock (lithology), the age of fossils and characterization of DNA (deoxyribonucleic acid). Biomarkers can be oligonucleotides, which are stretches of DNA molecules of simple fixed-length string.

DNA from fossils can form cross-links among themselves or with other molecules to the passage of time, hindering the use of
\end{abstract}


techniques such as PCR for study, therefore, in this work was applied to oligonucleotides a technique for determining the $K_{a}$ values (potential of the ionization constant), by UV spectrophotometry in the basic pH range, using as the model oligonucleotide d-AAAGAAA in $\mathrm{NaCl}$ aqueous solution, titrating it with $\mathrm{NaOH}$ solution. Also in the presence of a pH buffer solution $\left(\mathrm{NaHCO}_{3}\right)$. Furthermore pK values were determined for the same compound at different conditions of temperature, salts and mixtures of alcohol-water as solvent. We also assessed the $p K_{a}$ oligonucleotides $d-C C C G C C C$ and $d-A A G A A$.

$p K_{a}$ values were obtained on a range between 9 and 12. These results are a contribution to the knowledge of the physical properties of oligonucleotides, such data may be particularly useful in the aforementioned case of difficulty using other techniques such as PCR for the characterization and interpretation of oligonucleotides as biomarkers.

Keywords: oligonucleotides, biomarkers, potential of ionization constants.

\section{Introducción}

Los Marcadores biológicos o biomarcadores, son complejos moleculares que pueden estar presentes en fósiles, procedentes de biomoleculas de los organismos vivos. Debido a sus características generales son resistentes a la intemperie, la biodegradación, la evaporación y otros procesos biológicos por lo que comúnmente se conservan en las rocas y pueden ser utilizados para obtener información sobre la materia orgánica presente en las rocas fuente, la presencia de petróleo, las condiciones ambientales durante su sedimentación (diagénesis), la madurez térmica experimentada por el petróleo y/o roca (catagénesis), el grado de biodegradación, algunos aspectos de la mineralogía de la roca fuente (litología), la edad de los fósiles y en caracterización de DNA (Acido Desoxirribonucleico). Los biomarcadores pueden ser oligonucleótidos, que son fragmentos de moléculas de DNA de cadena sencilla de determinada longitud.

Se ha detectado que el DNA de fósiles puede conservarse hasta cerca de 500,000 años, pero puede formar enlaces cruzados entre si o con otras moléculas al paso del tiempo, dificultando el uso de técnicas como el PCR para su estudio (Peters et al., 2005), por ello, en el presente trabajo se aplicó a los oligonucleótidos, la técnica de determinación de los valores de los potenciales de las constantes de ionización $\left(\mathrm{pK}_{\mathrm{a}}\right)$, mediante espectrofotometría de luz ultravioleta en el intervalo básico de $\mathrm{pH}$, para diferentes oligonucleótidos.

El comportamiento químico y biológico del ácido desoxirribonucleico, DNA, está en gran medida definido por sus propiedades físicas. Un aspecto de suma importancia en esta macromolécula es su carácter de polianión. Cada cadena del DNA esta conformada por una hebra que contiene grupos desoxirribosa y grupos fosfato en estricta alternancia, donde los grupos desoxirribosa contienen además en derivación grupos heterocíclicos (timina, guanina, adenina y citosina). Como lo muestra la Figura 1, el puente de tipo diester fosfórico ocurre en cada una de las unidades nucleotídicas que constituyen la hebra, lo que confiere al DNA una alta densidad lineal de carga eléctrica, ya que cada grupo fosfato contiene, por ionización, una carga negativa. Este carácter de polianión explica muchas de las propiedades del DNA, su alto grado de solubilidad en solución acuosa, su facilidad para precipitar con alcohol, su electroforesis en campos eléctricos, la unión de cationes sobre la columna de DNA de doble hélice, así como la rigidez de esta última estructura (Sinden, 1994).

Aparte de esta ionización de los fosfatos, ocurren transiciones de protonación o desprotonación en las bases heterocíclicas del DNA, que se dan en ambientes ácidos o básicos y que causan cargas eléctricas positivas o negativas, adicionales a las cargas negativas de los fosfatos. Los valores aproximados de $\mathrm{pK}_{\mathrm{a}}$ que rigen estas transiciones están indicadas en la Figura 1. Este tipo de cargas también influye en las propiedades físicas y en la reactividad del DNA. En ambiente ácido se puede dar la protonación de las bases citosina (C), adenina (A) y guanina (G), lo que resulta localmente en la formación de cargas positivas, mientras que debido al carácter moderadamente ácido de las bases timina (T) y guanina $(\mathrm{G})$, estos grupos pueden ceder, en $\mathrm{pH}$ alcalino, a los hidrógenos de las posiciones $\mathrm{N}_{3}$ en la timina y de la $\mathrm{N}_{1}$ en la guanina (Saenger, 1984), experimentando la perdida de este hidrogeno (desprotonación), se obtiene la formación de cargas negativas (ionización) de las mencionadas bases. Por ello a estos compuestos se les pueden determinar valores de $\mathrm{pK}_{\mathrm{a}}$ o potencial de la constante de ionización. Para el ambiente acuoso, el $\mathrm{pK}_{\mathrm{a}}$ para una transición de una molécula en la forma no ionizada a la forma ionizada es numéricamente igual al valor de $\mathrm{pH}$ en que esta transición se da al 50\% (Popov et al., 2006). La fuerza iónica (I) de la solución se calcula para el punto medio de la transición, usando la fórmula:

$$
\mathrm{I}=\left(\sum_{\mathrm{i}} \mathrm{z}_{\mathrm{i}}^{2} \mathbf{c}_{\mathrm{i}}\right) / 2
$$

donde $z_{i}$ es la carga eléctrica del ión i, c es su concentración molal, y la suma considera todos los tipos de aniones y cationes (Klotz, 1964).

A pesar de la importancia de este fenómeno para el entendimiento de los ácidos nucléicos, se ha prestado escasa atención a la determinación cuantitativa de la propensión de los grupos involucrados a estas transiciones, es decir a la medición de los valores de los potenciales de las constantes de ionización $\left(\mathrm{pK}_{\mathrm{a}}\right)$ de estos procesos. Se sabe de manera general que estos valores de $\mathrm{pK}_{\mathrm{a}}$ para las bases heterocíclicas dentro de los ácidos nucléicos tienen valores semejantes a 


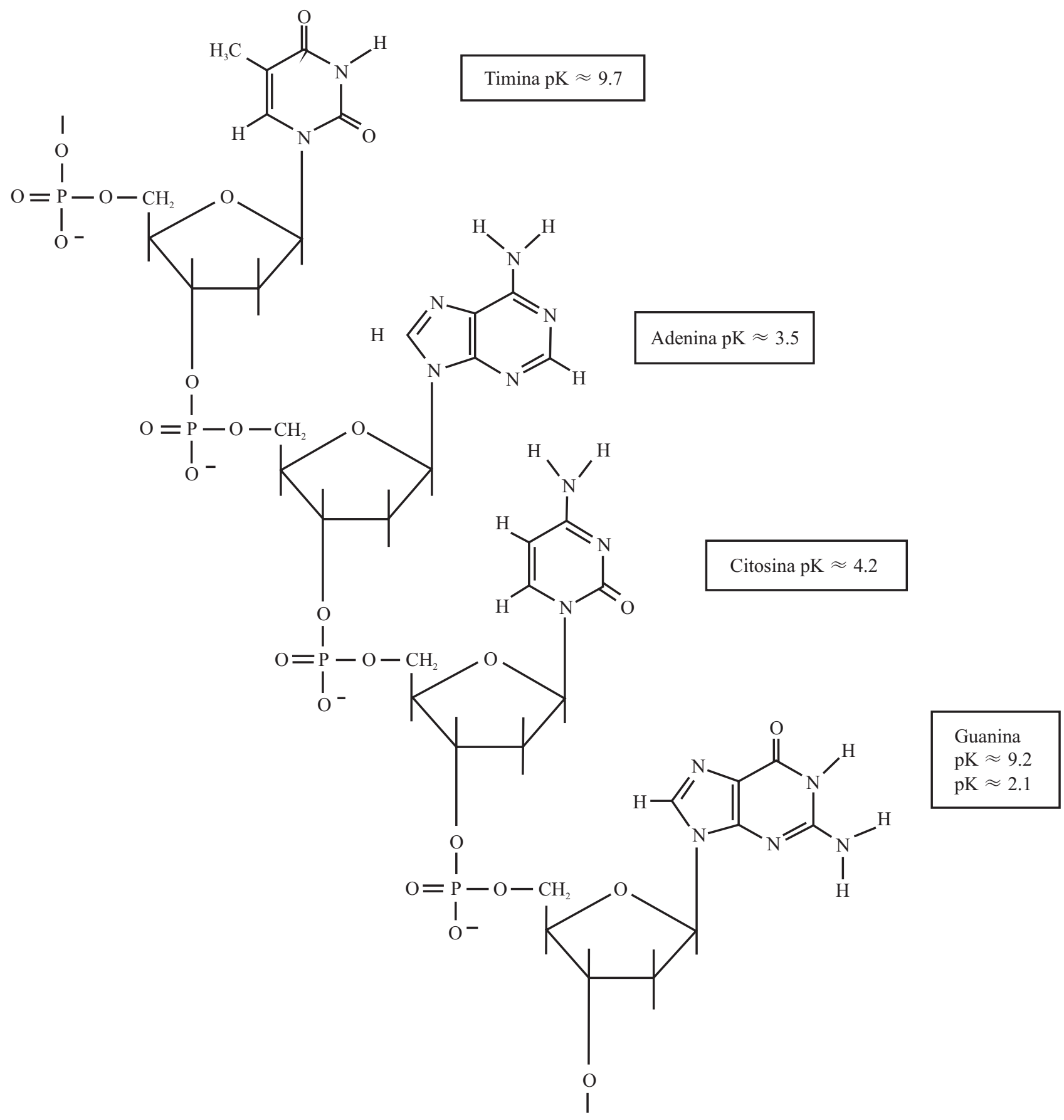

Figura 1: Estructura química de un tramo de DNA, con indicación de valores de $\mathrm{pK}_{\mathrm{a}}$ para los diferentes grupos (adaptado de Sinden, 1994).

los valores de $\mathrm{pK}_{\mathrm{a}}$ que tienen las correspondientes moléculas formadas por la base heterocíclica y el azúcar desoxirribosa (nucleósidos libres), que son de de 9.2 y 9.8 respectivamente para la desprotonación de la guanosina y la timidina, respectivamente, aunque recorridos hacia valores mayores, debido al efecto de campo eléctrico creado por las cargas negativas de los grupos fosfato, densamente alineados a lo largo de la macromolécula (Bloomfield et al., 2000). Pero se carece de un conocimiento cuantitativo y profundo de los valores de $\mathrm{pK}_{\mathrm{a}}$ para las bases dentro de los oligonucleótidos.

A raíz de estas consideraciones, el uso de las propiedades fisicoquímicas de los oligonucleótidos como biomarcadores, particularmente la absorbancia a determinadas longitudes de onda de las especies ionizadas y no ionizadas de guanina o timina en ácidos nucléicos de longitud significativa, sus valores de potencial de constantes de ionización, $\left(\mathrm{pK}_{\mathrm{a}}\right)$ se presenta como un proyecto de gran utilidad. Sobre todo en la situación ya mencionada que no se pueda emplear alguna 
otra técnica común de caracterización de DNA, como el caso del PCR (Peters et al., 2005).

\section{Metodología y materiales}

En general las titulaciones para determinar los valores de $\mathrm{pK}_{\mathrm{a}}$ para cada caso se llevaron a cabo en celdas de cuarzo de $1 \mathrm{~mL}$ o de $4 \mathrm{~mL}$, con paso óptico de $1 \mathrm{~cm}$, usando un espectrofotómetro Perkin Elmer Lambda 35, de doble haz, con capacidad de control de temperatura, mediante un baño maría. Durante las mediciones de absorbancia (A), la concentración del oligonucleótido es del orden de $10^{-5}$ $\mathrm{M}$ (en oligonucleótido). El barrido de $\mathrm{pH}$ se efectúa, ya sea en agua pura o en solución de amortiguador (bicarbonato de sodio), por adición de pequeños volúmenes de solución de $\mathrm{NaOH}$ valorada y mezclado con un microimán. Para control de masas, la celda con su contenido se pesó con una balanza analítica tras cada adición de solución. A lo largo de la titulación, el pH se calcula sobre la base del contenido inicial de amortiguador inicial y del total de $\mathrm{NaOH}$ agregado; también se midió directamente dentro de la cubeta con un microelectrodo de $\mathrm{pH}$ (Orion Modelo 05711 46). Para cada nuevo valor de $\mathrm{pH}$, se registra en duplicado la absorción de la solución a ocho diferentes longitudes de onda $(\lambda)$ entre 230 y $320 \mathrm{~nm}$.

Para la evaluación de los resultados, se grafican, en función del $\mathrm{pH}$, valores de razones de absorbancias medidas a diferentes longitudes de onda, para obtener la indicación más clara de la transición (ionización por desprotonación de la guanina contenida dentro del oligonucleótido). El valor de $\mathrm{pK}_{\mathrm{a}}$ para la desprotonación es el valor de $\mathrm{pH}$ para el cual el cambio de $\left(\mathrm{A}_{\lambda=270} / \mathrm{A}_{\lambda=255}\right)$ inicial a $\left(\mathrm{A}_{\lambda=270} / \mathrm{A}_{\lambda=255}\right)$ final se ha cumplido al 50\% (Clauwaert y Stockx, 1968; Acharya et al., 2002 y 2003; Airoldi et al., 2003).

El seguimiento de la desprotonación de la guanina por medio de espectrofotometría de luz ultravioleta, se basa en el hecho de que el valor de la absorbancia de la base heterocíclica cambia en medida sustancial al pasar de la forma no ionizada a la forma desprotonizada.

Las adeninas, que son las bases mayoritarias dentro de nuestros oligonucleótidos modelo, absorben fuertemente en el intervalo UV, pero su contribución es constante a lo largo de la titulación de $\mathrm{pH}$, ya que no sufren desprotonación.

Los detalles experimentales para cada caso de estudio se describen a continuación.

2.1 Determinación del $\mathrm{pK}_{\mathrm{a}}$ de la base guanina en el oligonucleótido d- $\mathrm{A}_{3} \mathrm{GA}_{3}$ en dependencia de la fuerza iónica

Esta determinación se realizó en dos condiciones:

\subsubsection{En solución acuosa sin amortiguador de $\mathrm{pH}$}

El oligonucleótido a estudiar se disuelve a una concentración $10^{-5} \mathrm{M}$, la misma solución contiene $\mathrm{NaCl}$ en una concentración $0 \mathrm{M}, 0.1 \mathrm{M}$ o $1 \mathrm{M}$ dentro de la celda espectrofotométrica provista de un microimán cubierto con teflón y una tapa hermética de teflón, la celda espectrofotométrica tiene un paso óptico de $1 \mathrm{~cm}$, en la solución el oligonucleótido tiene absorbancia máxima a longitudes de onda cercanas a $260 \mathrm{~nm}$, con valores aproximados de la unidad o ligeramente mayor (es decir que la solución absorbe al menos $90 \%$ de la potencia de la luz inicial a $260 \mathrm{~nm}$ ). Mediante el baño maría, la cubeta se mantiene a temperatura constante $\left(25^{\circ} \mathrm{C}\right.$ o la indicada) a lo largo de todo el experimento. La titulación de la solución de oligonucleótido dentro de la cubeta se efectúa mediante una serie de adiciones de volúmenes discretos de soluciones acuosas de $\mathrm{NaOH}$ a manera de cubrir el intervalo de $\mathrm{pH}$ entre $\mathrm{pH} 7$ y $\mathrm{pH}$ 13. Las soluciones estandarizadas de $\mathrm{NaOH}$ contienen $\mathrm{NaCl}$ a la misma concentración como la inicialmente seleccionada, para así mantener una concentración de ión sodio aproximadamente constante a través de la titulación. La adición de la solución estándar de $\mathrm{NaOH}$ a la cubeta se efectuó al exterior del espectrofotómetro (en volúmenes entre $1 \mu \mathrm{L}$ y $90 \mu \mathrm{L}$ ). Después de cada adición, la solución al interior de la cubeta fue mezclada brevemente por medio del microimán. La cubeta (con microimán, tapa y solución) se pesó en la balanza analítica, para verificar el volumen correcto de solución estándar de $\mathrm{NaOH}$. Con la cubeta dentro del espectrofotómetro, se registraron secuencialmente los valores de absorbancia a varias longitudes de onda discretas. Estas longitudes de onda para el oligonucleótido más frecuentemente usado (d-AAAGAAA), fueron las siguientes: $320 \mathrm{~nm}, 300 \mathrm{~nm}$, $280 \mathrm{~nm}, 275 \mathrm{~nm}, 270 \mathrm{~nm}, 265 \mathrm{~nm}, 260 \mathrm{~nm}$, y $255 \mathrm{~nm}$

La determinación de $\mathrm{pH}$ a lo largo de estas titulaciones se efectuó por medición continua de $\mathrm{pH}$ por microelectrodo.

En cuanto al tratamiento de los datos de absorbancia, los detalles son: se registran los valores de absorbancia a las diferentes longitudes de onda, luego se sustrae de cada uno de estos valores registrados, el valor de $\mathrm{A}_{\lambda=320}$. Esto sirve para compensar un posible corrimiento de la línea base común para las diferentes longitudes de onda que pudiera haber ocurrido durante el transcurso del experimento. El fundamento de esta estrategia es que el oligonucleótido no absorbe a longitudes de onda por encima de $305 \mathrm{~nm}$ (Saenger, 1984).

Se monitoreo la transición de desprotonación de la guanina en el oligonucleótido a través de cambios en la proporción entre los valores de absorbancia medidos a dos longitudes de ondas diferentes: $\mathrm{A}_{\lambda=255} / \mathrm{A}_{\lambda=260}$, las cuales se graficaron en función del $\mathrm{pH}$.

De las curvas de transición se obtiene el $\mathrm{pK}_{\mathrm{a}}$ para la guanina dentro del oligonucleótido, interpretándolas como gráficas de Hill. Esto se basa en la transición entre dos estados definidos (oligonucleótido con la guanina neutral y oligonucleótido con la guanina desprotonizada). Para los valores de las razones de absorbancia con que se monitorea la transición, se establece el valor que caracteriza la forma no ionizada del oligonucleótido (primer meseta 
de la curva), el cual es a un $\mathrm{pH}$ entre 7 y 9, así como el valor que caracteriza el oligonucleótido con la guanina enteramente desprotonizada (segunda meseta de la curva de transición), que se da aproximadamente entre $\mathrm{pH} 11$ y 12. (Figura 2), el valor de razón de absorbancias promedio de estas dos mesetas se da cuando un $50 \%$ de las guaninas están desprotonizadas, es decir, para ese valor exactamente intermedio del parámetro de seguimiento, el valor real de $\mathrm{pH}$ de la solución es idéntico al valor de $\mathrm{pK}_{\mathrm{a}}$ de la guanina en las condiciones y en el entorno oligonucleotídico empleado (Clauwaert y Stockx, 1968; Acharya et al., 2002 y 2003; Airoldi et al., 2003).

La fuerza iónica de la solución se calcula para el punto medio de la transición, usando la fórmula ya indicada de Klotz (1964)

\subsubsection{En solución amortiguadora de $\mathrm{pH}$ de bicarbonato de sodio}

Este tipo de experimento se efectúa de la misma manera que se describe en el punto anterior con algunos cambios. La diferencia central es que, al comenzar el experimento, la cubeta de muestra se carga con una solución que contiene, además de $\mathrm{NaCl}$ a la concentración deseada, $\mathrm{NaHCO}_{3}$ al $0.050 \mathrm{M}$. El ajuste de $\mathrm{pH}$ a través del experimento se efectúa nuevamente por adición de solución estándar de $\mathrm{NaOH}$ $(0.001 \mathrm{M}, 0.01 \mathrm{M}, 0.1 \mathrm{M}$ o $1 \mathrm{M})$, que además contiene $\mathrm{NaCl}$ en la concentración seleccionada, de manera que la concentración de esta sal queda constante a través del experimento.

El pH se evalúa por medición directa durante el experimento con el electrodo semimicro modelo 05711-
46, marca Orion. Las mediciones de $\mathrm{pH}$ se efectuaron a temperatura constante de $25^{\circ} \mathrm{C}$.

2.2 Estudio del efecto de diferentes temperaturas sobre el $\mathrm{pK}_{\mathrm{a}}$ de la base guanina incorporada en el oligonucleótido $\mathrm{d}-\mathrm{A}_{3} \mathrm{GA}_{3}$

El oligonucleótido d- $\mathrm{A}_{3} \mathrm{GA}_{3}$ se tituló con $\mathrm{NaOH}$, de la manera descrita en el punto 2.1.1, titulando en presencia de amortiguador de bicarbonato de sodio al 50 $\mathrm{mM}$, sin la adición de otra sal, a temperaturas de $10^{\circ} \mathrm{C}$, $25^{\circ} \mathrm{C}, 40^{\circ} \mathrm{C}, \mathrm{y} 55^{\circ} \mathrm{C}$. Los valores de $\mathrm{pH}$ se midieron a las temperaturas indicadas, que fueron mantenidas durante la corrida experimental mediante el baño maría acoplado al espectrofotómetro.

2.3 Estudio del efecto de diferentes sales sobre el $\mathrm{pK}_{\mathrm{a}}$ de la base guanina incorporada en el oligonucleótido d- $\mathrm{A}_{3} \mathrm{GA}_{3}$

El oligonucleótido d- $\mathrm{A}_{3} \mathrm{GA}_{3}$ se tituló con $\mathrm{NaOH}$ a $25^{\circ} \mathrm{C}$, en ausencia de amortiguador de $\mathrm{pH}$, de la manera descrita en el punto 2.1.1, con la excepción de que se ensayaron varias sales diferentes de $\mathrm{NaCl}$, las cuales fueron $\mathrm{LiCl}$ o $\mathrm{KCl}$ al $0.1 \mathrm{M}$, o $\mathrm{BaCl}_{2}$ al $0.05 \mathrm{M}$. Las soluciones estándar de $\mathrm{NaOH}$ que se añadieron durante la titulación contenían, además de la concentración deseada de $\mathrm{NaOH}$, la misma sal de la solución del oligonucleótido $\left(\mathrm{LiCl}, \mathrm{KCl} \circ \mathrm{BaCl}_{2}\right)$ a la concentración indicada, para que la concentración de esa sal fuera constante durante el experimento.

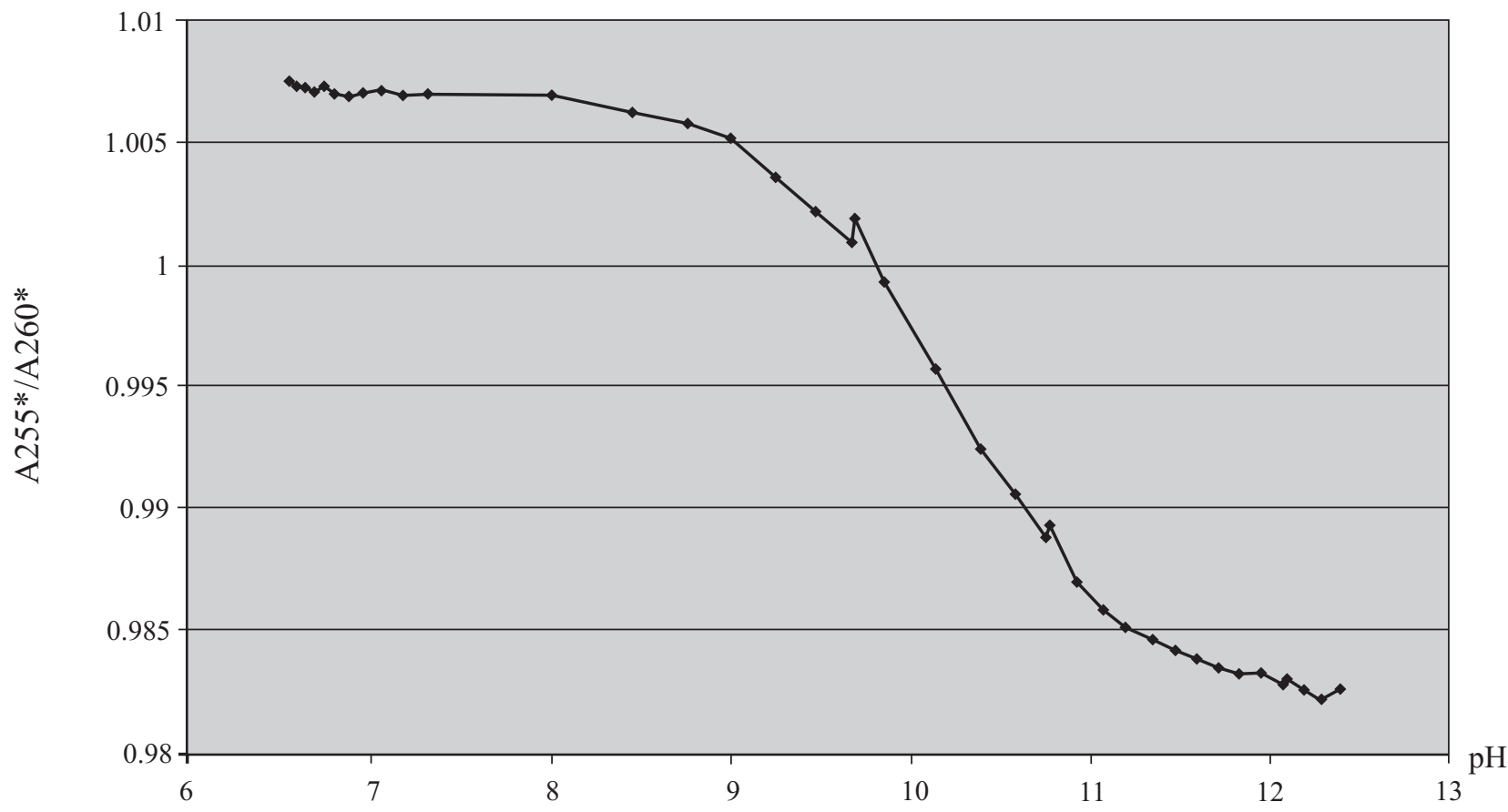

Figura 2: Determinación del valor de pKa en el punto medio de la curva sigmoidal (gráfica de Hill). 
2.4 Determinación del efecto del solvente sobre el $\mathrm{pK}_{\mathrm{a}}$ de la base guanina incorporada en el oligonucleótido d- $\mathrm{A}_{3} \mathrm{GA}_{3}$

El oligonucleótido d- $\mathrm{A}_{3} \mathrm{GA}_{3}$ se tituló con $\mathrm{NaOH}$ a $25^{\circ} \mathrm{C}$, sin amortiguador de $\mathrm{pH}$, sin otra sal añadida, en la forma descrita en el punto 2.1.1, con la excepción de que se usaron como solvente las mezclas agua/etanol $(3: 1, \mathrm{v}=$ volumen $/ \mathrm{v})$, agua/etanol $(1: 1, \mathrm{v} / \mathrm{v})$ o agua/etanol $(3: 7, \mathrm{v} / \mathrm{v})$. Las soluciones estándar de $\mathrm{NaOH}$ que se añadieron durante la titulación también fueron preparadas con estas mezclas de agua/etanol, de manera que la composición del solvente fue constante durante el experimento. No se llevaron a cabo mediciones de $\mathrm{pH}$ durante estos experimentos, ya que el concepto de $\mathrm{pH}$ aplica solo a soluciones acuosas.

2.5 Estudio del efecto de adición de bases de guanina sobre el $\mathrm{pK}_{\mathrm{a}}$ promediado de las mismas cuando están incorporadas en serie en el oligonucleótido

Estos experimentos se llevaron a cabo por el método descrito en el punto 2.1.2, titulando los oligonucleótidos d-AAAGAAA, d-AAAGGAAA, y d-AAAGGGAAA con $\mathrm{NaOH}$ a $25^{\circ} \mathrm{C}$ en solución amortiguadora de bicarbonato de sodio al $50 \mathrm{mM}$, sin añadidura de otra sal.

2.6 Estudio del efecto de la longitud total del oligonucleótido sobre el $\mathrm{pK}_{\mathrm{a}}$ de la base guanina incorporada en el oligonucleótido

Para este experimento se compararon los valores de $\mathrm{pK}_{\mathrm{a}}$ de la base guanina ubicada en posición central en los oligonucleótidos d-AAGAA y d-AAAGAAA, titulando como en el punto 2.1 .1 , a $25^{\circ} \mathrm{C}$ con $\mathrm{NaOH}$, sin amortiguador de $\mathrm{pH}$ y sin otra sal.

2.7 Estudio del efecto de bases diferentes de adenina como entorno de la guanina incorporada en el oligonucleótido

Para este experimento se compararon los valores de $\mathrm{pK}_{\mathrm{a}}$ de la base guanina en los oligonucleótidos d-AAAGAAA y d-CCCGCCC, titulando como se describe, en el punto 2.1.1, a $25^{\circ} \mathrm{C}$ con $\mathrm{NaOH}$, sin amortiguador de $\mathrm{pH}$ y sin otra sal.

2.8 Materiales Estudiados

Oligonucleótidos:

d-AAGAA, d-AAAGAAA, d-AAAGGAAA, d-AAAGGGAAA, d-CCCGCCC

\section{Resultados}

A continuación se presentan los valores de potenciales de las constantes de ionización obtenidos.
3.1 Determinación del $\mathrm{pK}_{\mathrm{a}}$ del grupo guanina en el oligonucleótido d- $\mathrm{A}_{3} \mathrm{GA}_{3}$

\subsubsection{En solución acuosa sin amortiguador de $p H$ :}

La tabla 1 contiene los valores de $\mathrm{pK}_{\mathrm{a}}$ del oligonucleótido d-AAAGAAAA en las condiciones descritas

Tabla 1. Valores de $\mathrm{pK}_{\mathrm{a}}$ determinados para d-AAAGAAA en solución acuosa sin amortiguador de $\mathrm{pH}$.

\begin{tabular}{ccc}
\hline $\mathrm{NaCl}$ & $\mathrm{I}$ & $\mathrm{pK}_{\mathrm{a}}$ \\
$(\mathrm{M})$ & (a media transición) & $($ a 25ㅇ) \\
\hline 0 & 0.0007 & 10.82 \\
0.1 & 0.1002 & 10.39 \\
1 & 1.0001 & 10.00 \\
\hline
\end{tabular}

3.1.2 En solución amortiguadora de $\mathrm{pH}$ de bicarbonato de sodio al $0.05 \mathrm{M}$.

La tabla 2 resume los resultados obtenidos para el $\mathrm{pK}_{\mathrm{a}}$ de la guanina en el oligonucleótido d-AAAGAAA.

Tabla 2. Valores de $\mathrm{pK}_{\mathrm{a}}$ determinados para d-AAAGAAA en solución acuosa con $\mathrm{NaHCO}_{3}(0.05 \mathrm{M})$ como amortiguador de $\mathrm{pH}$.

\begin{tabular}{ccc}
\hline $\mathrm{NaCl}$ & $\mathrm{I}$ & $\mathrm{pK}_{\mathrm{a}}$ \\
$(\mathrm{M})$ & $($ a media transición) & $\left(\mathrm{a} 25^{\circ} \mathrm{C}\right)$ \\
\hline 0 & 0.095 & 10.84 \\
0.1 & 0.193 & 10.73 \\
0.3 & 0.392 & 10.69 \\
1 & 1.087 & 10.43 \\
\hline
\end{tabular}

3.2 Estudio del efecto de la temperatura sobre el $\mathrm{pK}_{\mathrm{a}}$ del oligonucleótido d- $\mathrm{A}_{3} \mathrm{GA}_{3}$

Los resultados se muestran en la tabla 3.

Tabla 3. Valores de $\mathrm{pK}_{\mathrm{a}}$ determinados para d-AAAGAAA en $\mathrm{NaHCO}_{3}$ al $0.05 \mathrm{M}$ como solución amortiguadora, a diferentes temperaturas.

\begin{tabular}{ccc}
\hline $\mathrm{T}\left({ }^{\circ} \mathrm{C}\right)$ & $\mathrm{I}$ & $\mathrm{pK}_{\mathrm{a}}$ \\
& (a media transición) & \\
\hline 10 & 0.0971 & 10.95 \\
25 & 0.0947 & 10.84 \\
40 & 0.0913 & 10.60 \\
55 & 0.0572 & 9.32 \\
\hline
\end{tabular}

3.3 Estudio del efecto de diferentes sales sobre el $\mathrm{pK}_{\mathrm{a}}$ del oligonucleótido d- $\mathrm{A}_{3} \mathrm{GA}_{3}$

En la Tabla 4 se presentan los resultados de estas pruebas. 
Tabla 4. Valores de $\mathrm{pK}$ determinados para d-AAAGAAA en presencia de diferentes sales, a $25^{\circ} \mathrm{C}$.

\begin{tabular}{ccc}
\hline $\mathrm{Sal}(0.1 \mathrm{M})$ & $\mathrm{I}$ & $\mathrm{pK}_{\mathrm{a}}$ \\
& (a media transición) & $\left(\mathrm{a} 25^{\circ} \mathrm{C}\right)$ \\
\hline $\mathrm{LiCl}$ & 0.101 & 10.63 \\
$\mathrm{NaCl}$ & 0.100 & 10.62 \\
$\mathrm{KCl}$ & 0.102 & 10.87 \\
$\mathrm{BaCl}_{2}$ & 0.151 & 10.41 \\
\hline
\end{tabular}

3.4 Determinación del efecto del solvente sobre el $\mathrm{pK}_{\mathrm{a}}$ del oligonucleótido d- $\mathrm{A}_{3} \mathrm{GA}_{3}$

Los resultados se indican en la tabla 5 .

Tabla 5. Valores de pKa determinados para d-AAAGAAA en presencia de alcohol etílico a diferentes concentraciones, a $25^{\circ} \mathrm{C}$.

\begin{tabular}{cc}
\hline Solvente & $(\mathrm{NaOH})$ libre $(\mathrm{M})$ \\
& $($ a media transición $)$ \\
\hline agua & 0.0030 \\
agua/etanol $(75: 25, \mathrm{v} / \mathrm{v})$ & 0.0029 \\
agua/etanol $(50: 50, \mathrm{v} / \mathrm{v})$ & 0.0013 \\
agua/etanol $(30: 70, \mathrm{v} / \mathrm{v})$ & 0.0017 \\
\hline
\end{tabular}

3.5 Estudio del efecto del número de bases guanina presentes en el oligonucleótido sobre el $\mathrm{pK}_{\mathrm{a}}$ del mismo.

Los resultados se encuentran listados en la tabla 6 .

Tabla 6. Valores de $\mathrm{pK}_{\mathrm{a}}$ determinados para varios oligonucleótidos de la forma d-AAAGnAAA en solución acuosa de $\mathrm{NaHCO}_{3}$ al $0.05 \mathrm{M}$, a $25^{\circ} \mathrm{C}$.

\begin{tabular}{ccc}
\hline Oligonucleótido & $\mathrm{I}$ & $\mathrm{pK}_{\mathrm{a}}$ \\
& (a media transición) & $\left(\mathrm{a} 25^{\circ} \mathrm{C}\right)$ \\
\hline d-AAAGAAA & 0.095 & 10.84 \\
d-AAAGGAAA & 0.099 & 11.15 \\
d-AAAGGGAAA & 0.087 & 10.47 \\
\hline
\end{tabular}

3.6 Estudio del efecto de bases diferentes de adenina como entorno de la guanina incorporada en el oligonucleótido

Los resultados de esta titulación se presentan en la tabla 7.

Tabla 7. Valores de $\mathrm{pK}_{\mathrm{a}}$ determinados para oligonucleótidos de la forma d- $\mathrm{X}_{3} \mathrm{GX}_{3}$ en solución acuosa sin sal, a $25^{\circ} \mathrm{C}$.

\begin{tabular}{ccc}
\hline Oligonucleótido & $\mathrm{I}$ & $\mathrm{pK}_{\mathrm{a}}$ \\
& (a media transición) & (a 25 $\left.{ }^{\circ} \mathrm{C}\right)$ \\
\hline d-AAAGAAA & 0.00070 & 10.82 \\
d-CCCGCCC & 0.00012 & 10.07 \\
\hline
\end{tabular}

3.7 Estudio del efecto de la longitud total del oligonucleótido sobre su $\mathrm{pK}_{\mathrm{a}}$

Los resultados se muestran en la tabla 8 .

Tabla 8: Valores de $\mathrm{pK}_{\mathrm{a}}$ determinados para oligonucleótidos de la forma d- $A_{n} G_{n}$ en solución acuosa sin sal a $25^{\circ} \mathrm{C}$.

\begin{tabular}{ccc}
\hline Oligonucleótido & $\mathrm{I}$ & $\mathrm{pK}_{\mathrm{a}}$ \\
& (a media transición) & (a 25ํ) \\
\hline d-AAAGAAA & 0.0007 & 10.82 \\
d-AAGAA & 0.0003 & 10.17 \\
\hline
\end{tabular}

\section{Discusión}

4.1 Determinación del $\mathrm{pK}_{\mathrm{a}}$ del grupo guanina en el oligonucleótido d- $\mathrm{A}_{3} \mathrm{GA}_{3}$

\subsubsection{En solución acuosa sin amortiguador de pH}

En los resultados mostrados en la tabla 1 se observa que cuando la solución con oligonucleótido posee mayor fuerza iónica, al oligonucleótido se le determina un menor valor de $\mathrm{pK}_{\mathrm{a}}$ de la guanina, resultado que se espera, basado en un mayor apantallamiento de la carga negativa de los fosfatos por la creciente concentración de iones de sodio, lo que reduce el efecto de campo de los fosfatos sobre las bases nitrogenadas (Clauwaert y Stockx, 1968), facilitándose de esta manera la desprotonación de las guaninas.

\subsubsection{En solución amortiguadora de pH de bicarbonato de sodio}

Analizando los resultados de la tabla 2 se observa también, como en el primer caso, que a mayor fuerza iónica de la solución con oligonucleótido se obtiene menor valor de $\mathrm{pK}_{\mathrm{a}}$ de la guanina, pero dentro de un rango mas reducido debido al efecto regulador del $\mathrm{pH}$ del $\mathrm{NaHCO}_{3}$ en la solución.

4.2 Estudio del efecto de la temperatura sobre el $\mathrm{pK}_{\mathrm{a}}$ del oligonucleótido d- $\mathrm{A}_{3} \mathrm{GA}_{3}$

El efecto de la temperatura sobre la acidez guanina en el entorno oligonucleotídico se estudió en el intervalo de $10^{\circ} \mathrm{C}$ a $55^{\circ} \mathrm{C}$, en solución acuosa amortiguada con 0.05 $\mathrm{M}$ de bicarbonato de sodio, sin otra sal añadida. En los resultados que se muestran en la tabla 3 , se observa que entre $10^{\circ} \mathrm{Cy} 40^{\circ} \mathrm{C}$ se da una reducción paulatina y moderada del $\mathrm{pK}_{\mathrm{a}}$, es decir un incremento en la acidez de la guanina, como es de esperar para un ácido del tipo N-H al aumentar la temperatura (Bloomfield et al., 2000). El cambio que se presenta entre $40^{\circ} \mathrm{C}$ y $55^{\circ} \mathrm{C}$, es un corrimiento de $\mathrm{pK}_{\mathrm{a}}$ ampliamente mayor a una unidad de $\mathrm{pH}$. 
4.3 Estudio del efecto de diferentes sales sobre el $\mathrm{pK}_{\mathrm{a}}$ del oligonucleótido d- $\mathrm{A}_{3} \mathrm{GA}_{3}$

Se puede observar en la tabla 4 que a menor tamaño del catión disminuye el valor del $\mathrm{pK}_{\mathrm{a}}$ y a mayor carga del catión se obtiene el mismo efecto, reducción en el valor del $\mathrm{pK}_{\mathrm{a}}$ del oligonucleótido, este efecto puede deberse en ambos casos a la mayor facilidad (por parte de un catión pequeño y un catión divalente) para apantallar el efecto de campo eléctrico del polianión fosfato sobre el proceso de ionización del oligonucleótido.

4.4 Determinación del efecto del solvente sobre el $\mathrm{pK}_{\mathrm{a}}$ del oligonucleótido d- $\mathrm{A}_{3} \mathrm{GA}_{3}$

En este caso como ya se había mencionado, por no tratarse de una solución acuosa, no se aplica el concepto de $\mathrm{pH}$ sino que se maneja concentración de $\mathrm{NaOH}$ libre a media transición. Los resultados de la tabla 5 indican que no hay diferencia en la concentración de $\mathrm{NaOH}$ libre a media transición para soluciones en $100 \%$ de agua y en agua/etanol $(3: 1, \mathrm{v} / \mathrm{v})$, mientras que para soluciones de agua/ etanol $(1: 1, \mathrm{v} / \mathrm{v})$ o de agua/etanol $(3: 7, \mathrm{v} / \mathrm{v})$ la concentración de $\mathrm{NaOH}$ libre a media transición se reduce por un factor de aproximadamente 2, en ambos casos.

4.5 Estudio del efecto del número de bases guanina presentes en el oligonucleótido sobre el $\mathrm{pK}_{\mathrm{a}}$ del mismo

En la comparación de los resultados listados en la tabla 6 para los oligonucleótidos d-AAAGAAA y d-AAAGGAAA se aprecia un aumento en el valor de $\mathrm{pK}_{\mathrm{a}}$ por 0.3 unidades. Es decir que para la serie de dos guaninas, la ionización de la primer $\mathrm{G}$ se ve afectada de manera negativa por la presencia de la segunda $\mathrm{G}$, es decir requiere de mayor basicidad en la solución (corrimiento del $\mathrm{pK}_{\mathrm{a}}$ hacia un valor mayor), comparado con la situación que se da con el oligómero d-AAAGAAA, mientras que la segunda disociación se ve tan desfavorecida que no se observó en la titulación, posiblemente reflejando la dificultad de formar varias guaninas desprotonizadas en serie. La introducción de una tercer guanina en serie redunda en un valor aparente de $\mathrm{pK}_{\mathrm{a}}$ que es decididamente menor al valor de $\mathrm{pK}_{\mathrm{a}}$ determinado para el caso de d-AAAGAAA; es decir, en la serie de tres grupos guanina al menos uno ostenta una acidez claramente mayor a las de las otras guaninas.

4.6 Estudio del efecto de bases diferentes de adenina como entorno de la guanina incorporada en el oligonucleótido

En los resultados de esta titulación que se presenta en la tabla 7 se observa en el menor valor de $\mathrm{pK}_{\mathrm{a}}$ del oligonucleótido d-CCCGCCC, una mayor facilidad del mismo para ionizarse (mayor acidez) en comparación del oligonucleótido d-AAAGAAA, producto de la presencia de la base citocina en el primero.

4.7 Estudio del efecto de la longitud total del oligonucleótido sobre su $\mathrm{pK}_{\mathrm{a}}$

Si la propensión a la desprotonación de la guanina dentro del entorno oligomérico está sujeta al efecto de campo eléctrico que proveen los grupos fosfato en su totalidad, es de esperar que el $\mathrm{pK}_{\mathrm{a}}$ medido para la base ionizable en posición central del oligómero dependa de la longitud del mismo. Para evaluar este posible efecto, se realizaron las pruebas en las condiciones más idóneas para detectarlo, es decir, a baja fuerza iónica, donde el apantallamiento de las cargas negativas de los grupos fosfato fuera mínimo. Para esto, se tituló el pentanucleótido d-AAGAA, el valor de $\mathrm{pK}_{\mathrm{a}}$ derivado de esta titulación es de 10.17 (tabla 8), claramente inferior al $\mathrm{pK}_{\mathrm{a}}$ determinado en condiciones idénticas para el heptámero correspondiente, d-AAAGAAA. Esto indica que la acidez de la guanina aumenta al disminuir el efecto de campo eléctrico producido por un menor número de grupos fosfato en el oligonucleótido.

En el presente estudio cabe destacar como valor más significativo de $\mathrm{pK}_{\mathrm{a}}$ obtenido, el de 10.8 correspondiente al oligonucleótido d-AAAGAAA a $25^{\circ} \mathrm{C}$ y fuerza iónica baja, por ser las condiciones más semejantes a las condiciones ambientales y constituirse por lo tanto en un valor referente en los diferentes estudios del presente trabajo.

De la misma forma, es importante subrayar que este proyecto de investigación es pionero en su tipo, sobre todo por la amplitud de variables investigadas que pueden influir sobre el fenómeno de ionización de oligonucleótidos en medio alcalino, no existiendo por lo tanto una amplitud de referencias similares con las cuales establecer una comparación y en consecuencia una mayor discusión de resultados.

\section{Conclusiones}

Los valores de $\mathrm{pK}_{\mathrm{a}}$ obtenidos varían entre 9 y 12 unidades. Estos resultados obtenidos aplicando a los oligonucleótidos la técnica de determinación de los valores de $\mathrm{pK}_{\mathrm{a}}$ (potencial de la constante de ionización), mediante espectrofotometría de luz ultravioleta en el intervalo básico de $\mathrm{pH}$, confirman como oligonucleótido modelo ideal el d-AAAGAAA. De igual manera los resultados obtenidos, son un aporte al conocimiento de las propiedades físicas de los oligonucleótidos, datos especialmente útiles cuando se dificulte el uso de otras técnicas como el PCR para la caracterización e interpretación de oligonucleótidos como biomarcadores. 


\section{Referencias}

Acharya, S., Acharya, P., Földesi, A., Chattopadhyaya, J., 2002, Cross Modulation of Physicochemical Character of Aglycones in Dinucleoside (3'-5') Monophosphates by the Nearest Neighbor Interaction in the Stacked State: Journal of the American Chemical Society, 124, 13722-13730.

Acharya, P., Acharya, S., Földesi, A., Chattopadhyaya, J., 2003, Tandem Electrostatic Effect from the First to the Third Aglycon in the Trimeric RNA Owing to the Nearest Neighbor Interaction: Journal of the American Chemical Society, 125, 2094-2100.

Airoldi, M., Boicelli, C.A., Gennaro, G., Giomini, M., Giuliani, A.M., Giustini M., 2003, Titration of Poly(dA-dT)•Poly(dA-dT) in Solution at Variable $\mathrm{NaCl}$ Concentration: Biopolymers, 75, 118-127.

Bloomfield, V.A., Crothers, D.M., Tinoco, I., 2000, Nucleic Acids: Structure, Properties and Functions: Sausalito, Cal. U.S.A., University Science Books.

Clauwaert, J., Stockx, J., 1968, Acid Denaturation of PolyadenylicPolyuridylic Complexes: Verlag der Zeitschrift für Naturforschung, 23b, 25-30.
Klotz, I.M., 1964, Chemical Thermodynamics: Amsterdam, W.A. Benjamin, Inc. New York.

Peters, K.E., Walters, C.C., Moldowan, J.M., 2005, The Biomarker Guide, Volume I: Biomarkers and isotopes en the environment and the human history: Cambridge University Press, United Kingdom.

Popov, K., Ronkkomaki, H., Lajunen, L.H.J., 2006, Guidelines for NMR Measurements for Determination of High and Low $\mathrm{pK}_{\mathrm{a}}$ Values. Pure and Applied Chemistry, 78(3), 663-675.

Saenger, W., 1984, Principles of Nucleic Acid Structure: Springer-Verlag New York Inc. U.S.A.

Sinden, R.R., 1994, DNA Structure and Function: Academic Press, San Diego.

Manuscrito recibido: Febrero 27, 2009.

Manuscrito corregido recibido: Junio 11, 2009.

Manuscrito aceptado: Junio 16, 2009. 